\title{
Multilayer brain network combined with deep convolutional neural network for detecting major depressive disorder
}

\author{
Weidong Dang · Zhongke Gao · Xinlin Sun - Rumei Li • Qing Cai · \\ Celso Grebogi
}

Received: date / Accepted: date

\begin{abstract}
As a global and grievous mental disease, major depressive disorder (MDD) has received much attention. Accurate detection of MDD via physiological signals represents an urgent research topic. Here, a frequencydependent multilayer brain network, combined with deep convolutional neural network (CNN), is developed to detect the MDD. Multivariate pseudo Wigner distribution is firstly introduced to extract the time-frequency characteristics from the multi-channel EEG signals. Then multilayer brain network is constructed, with each layer corresponding to a specific frequency band. Such multilayer framework is in line with the nature of the workings of the brain, and can effectively characterize the brain state. Further, a multilayer deep CNN architecture is designed to study the brain network topology features, which is finally used to accurately detect MDD. The experimental results on a publicly available MDD dataset show that the proposed approach is able to detect MDD with state-of-the-art accuracy of $97.27 \%$. Our approach, combining multilayer brain network and deep CNN, enriches the multivariate time series analysis theory and helps to better characterize and recognize the complex brain states.
\end{abstract}

Keywords Electroencephalogram - Major depressive disorder · Complex network · Convolutional neural network

W. Dang · Z. Gao $(*) \cdot$ X. Sun · R. Li . Q. Cai

School of Electrical and Information Engineering, Tianjin University, Tianjin 300072, China

Tel.: +86-15022142526

E-mail: zhongkegao@tju.edu.cn

C. Grebogi

Institute for Complex Systems and Mathematical Biology, Kings College, University of Aberdeen, Aberdeen AB24 3UE, UK

\section{Introduction}

Major depressive disorder (MDD) has become a worldwide mental illness for people of all ages [1, 2]. According to a survey listed in [3], the percentage of MDD patients in Chinese children and adolescents was about $1.3 \%$ in 2018 . Globally, more than 300 million people suffer from depression. Unlike slight changes of mood in daily life, MDD may lead to a large number of serious health problems. More seriously, MDD patients have a higher suicidal tendency than healthy people. Therefore, accurate and timely detection of MDD is of great significance in scientific research and social life.

In the past few years, related research $[4,5]$ has gained a lot of attention. The analysis of MDD using electrophysiological signals has become a hot topic. Various electrophysiological signals, such as functional magnetic resonance imaging (fMRI) [6, 7], electrocardiogram (ECG) $[8,9]$, electroencephalogram (EEG) [1014], etc. have been adopted. Among them, EEG possesses the advantage of easy access, low cost, non-invasive, and high temporal resolution. It is favored in the study of MDD [15]. For example, Goldschmied et al. [16] analyzed the changes of EEG in the theta band and pointed out that sleep slow-wave activity played a whole different role in the regulation of sleep stability between healthy people and MDD patients. Murphy et al. [17] studied the impact of the severity of depression on working memory maintenance from the view of EEG analysis. In particular, given the complexity of the brain, EEG is characterized by its nonlinear and non-stationary nature [18]. Effective information from the frequency or time domains is constantly changing, and affects each other. Combining time and frequency information to analyze EEG signals can provide a more comprehensive understanding of the brain. Existing studies have shown 
that time-frequency analysis, developed from the traditional frequency domain analysis, can exactly provide the necessary theoretical support for this [19, 20]. Tian et al. [21] used short-time Fourier transform (STFT) to extract the 2-D feature of motor imagery EEG signals, and sent it into a convolutional neural network for binary classification. Bhattacharyya et al. [22] decomposed the EEG signal into rhythm signals of different frequency bands through empirical wavelet transform (EWT), and used them to detect the epileptic foci. Recently, multivariate pseudo Wigner distribution (MPWD) has been successfully applied to emotion detection based on multichannel EEG signals due to its lower computational cost [23], and it has shown the great potential in multivariate time-frequency analysis.

In recent years, complex network derived from complex systems has evolved into an effective theory for analyzing complex dynamics. A typical complex network topology consists of nodes and edges. For a specific research topic, one should elaborately choose the nodes. Then the complex system characteristics can be mapped into the network topology, namely, the edge relationship among these nodes. Complex network analysis has been involved in many fields and has achieved great successes [24-26]. Human brain is recognized as an extremely complex and fascinating system. The application of complex network theory to brain research has gained a lot of attention, especially in multi-channel EEG signal analysis [27, 28]. Functional brain network is a typical example, where brain electrodes are set as nodes and then the edges can be determined via diverse correlation measures between electrodes. Commonly used measures include coherence, mutual information, phase lag index, phase locking value (PLV) $[29,30]$, and others. Among them, taking nonlinearity into account, PLV gives the modulus of the averaged instantaneous phase differences between two studied time series and is employed in this work. Moreover, complex system exhibits obvious multiple characteristics, so does the brain. As the latest development in complex networks, multilayer network [31-34] possesses large number of nodes in multiple layers with different types of edges. Different layers correspond to different aspects of the studied system and allow providing a more intuitive and accurate characterization. Plenty of successful applications have been achieved in diverse fields involving linguistic subsystem [32], epidemic spreading [34], multiphase flow [35, 36] and brain research [37].

Complex systems contain the evolution of complex patterns. The understanding of these complex patterns provides a feasible direction for solving various classification problems. Machine learning (ML) is such a popular approach to do this. Diverse machine learning algorithms involving feature extraction, feature selection, and classification have been widely used. The same is true in MDD research [10, 13]. In recent years, as a particular subset of ML methods, deep learning [30, 38-40] has achieved many state-of-the-art results in diverse fields like object detection, speech recognition, and natural language processing. Deep learning is an end-to-end learning method, can extract deeper and intrinsic representation directly from input by itself. So far, numbers of architectures of deep learning have been proposed, which also includes the development of convolution neural network (CNN). CNN allows extracting a variety of potential features from inputs by using many convolution kernels. Related models with more layers, more connections [41] and more detailed convolutions [42, 43] have been proposed for better results. All these confirm that CNN method is a good choice to dig the effective features for characterizing the studied systems.

In this paper, a novel framework combining multilayer network and deep learning is developed for recognition of MDD. We first perform MPWD on multichannel EEG signals to conduct time-frequency representation (TFR). Then taking the rhythm specificity into account, a novel frequency-dependent multilayer brain (FDMB) network is inferred, where each layer involves a PLV-based functional brain network, corresponding to one specific frequency band. Such FDMB network dose consider and balance both the frequency characteristics and channel coupling of the multi-channel EEG signals, providing a comprehensive mapping of the brain. Moreover, considering the strong ability of deep learning for structure feature learning, we develop a multilayer deep CNN with FDMB networks served as the inputs. The results show that such combination of multilayer network and deep learning performs well on MDD detection task and can achieve a state-of-the-art accuracy of $97.27 \%$. The whole structure of our work is shown in Fig. 1.

\section{Methodology}

\subsection{The construction of FDMB network}

Multi-channel EEG signals are a direct reflection of the brain, which presents an obvious frequency-dependent characteristics. Existing work has shown that different frequency bands are related to different brain functions. From the point of multi-frequency analysis, combined with the ability of complex network for characterizing complex topological features, FDMB network is developed here. The schematic diagram of FDMB network is 


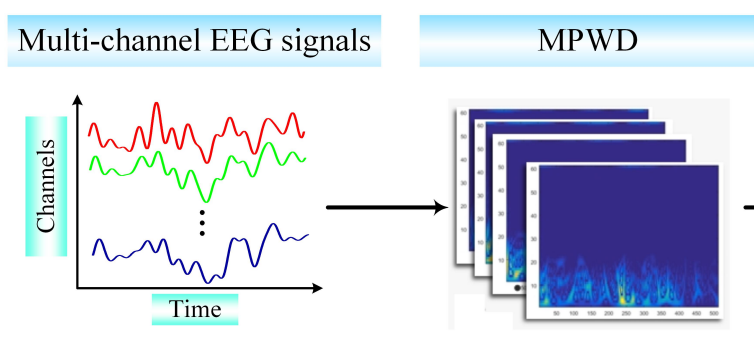

Fig. 1 The overall framework of our work.

shown in Fig. 1. Taking multi-channel time series $\mathbf{x}_{+}(t)$ as an example.

$\mathbf{x}_{+}(t)=\left[\begin{array}{c}a_{1}(t) e^{i \phi_{1}(t)} \\ a_{2}(t) e^{i \phi_{2}(t)} \\ \vdots \\ a_{p}(t) e^{i \phi_{p}(t)}\end{array}\right], t=1,2, \ldots, N$

where $N$ represents the length of the signals, $p$ denotes the number of channels, $a_{i}(t), i=1,2, \ldots, p$ and $\phi_{i}(t), i=1,2, \ldots, p$ represent the instantaneous amplitudes and phases, respectively. MPWD is first used to determine the time-frequency representation of $\mathbf{x}_{+}(t)$. MPWD allows effectively capturing the non-stationary and transient features, which exactly meets the need for EEG analysis. For $\mathbf{x}_{+}(t)$, Wigner distribution, also known as Wigner-Ville distribution, is defined as

$W D(\omega, t)=\int_{-\infty}^{\infty} \mathbf{x}_{+}^{H}(t-\tau / 2) \mathbf{x}_{+}(t+\tau / 2) e^{-j \omega \tau} d \tau$,

where $\mathbf{x}_{+}^{H}(t)$ is the Hermitian transpose of $\mathbf{x}_{+}(t)$. The inverse of Wigner distribution can be described by

$\mathbf{x}_{+}^{H}(t-\tau / 2) \mathbf{x}_{+}(t+\tau / 2)=\frac{1}{2 \pi} \int_{-\infty}^{\infty} W D(\omega, t) e^{j \omega \tau} d \tau$

The central frequency of the Wigner distribution for $\mathbf{x}_{+}(t)$ at time $t$ can be described by

$\langle\omega(t)\rangle=\frac{\int_{-\infty}^{\infty} \omega W D(\omega, t) d \omega}{\int_{-\infty}^{\infty} W D(\omega, t) d \omega}$.

By using the inverse Wigner distribution, (4) can be rewrote as the form of (5):

$$
\begin{aligned}
\langle\omega(t)\rangle & =\left.\frac{\frac{d}{j d \tau}\left[\mathbf{x}_{+}^{H}(t-\tau / 2) \mathbf{x}_{+}(t+\tau / 2)\right]_{\mid \tau=0}}{\mathbf{x}_{+}^{H}(t-\tau / 2) \mathbf{x}_{+}(t+\tau / 2)}\right|_{\mid \tau=0} \\
& =\frac{1}{2 j} \frac{\left[\mathbf{x}_{+}^{H}(t) \mathbf{x}^{\prime}(t)-\mathbf{x}_{+}^{\prime}(t) \mathbf{x}_{+}(t)\right]}{\mathbf{x}_{+}^{H}(t) \mathbf{x}_{+}(t)} .
\end{aligned}
$$

According to [23], [44], the joint instantaneous frequency of multivariate signals is

$\omega_{x}(t)=\frac{\Im\left\{\mathbf{x}_{+}^{H}(t) \frac{d}{d t} \mathbf{x}_{+}(t)\right\}}{\left\|\mathbf{x}_{+}(t)\right\|^{2}}=\frac{\sum_{i=1}^{N} a_{i}^{2}(t) \phi_{i}^{\prime}(t)}{\sum_{i=1}^{N} a_{i}^{2}(t)}$,

where the symbol $\Im$ stands for the imaginary part of a complex signal and $\phi_{i}^{\prime}(t)$ is the instantaneous frequency for each sub-component. In a similar way, the instantaneous bandwidth follows from

$v_{x}^{2}(t)=\frac{\int_{-\infty}^{\infty}\left(\omega-\omega_{x}(t)\right)^{2} W D(\omega, t) d \omega}{\int_{-\infty}^{\infty} W D(\omega, t) d \omega}$,

with

$\frac{1}{2 \pi} \int_{-\infty}^{\infty} \omega^{2} W D(\omega, t) d \omega$

$=-\frac{d^{2}}{d \tau^{2}}\left[\mathbf{x}_{+}^{H}(t-\tau / 2) \mathbf{x}_{+}(t+\tau / 2)\right]_{\mid \tau=0}$.

Finally, we use a multivariate extension of the pseudo Wigner distribution, where a window function is employed to evaluate (2), and therefore MPWD can be realized. More details see [23].

Then, six frequency bands, including delta $\delta(0.5-4$ $\mathrm{Hz})$, theta $\theta(4-8.5 \mathrm{~Hz})$, alpha $\alpha(8.5-17.5 \mathrm{~Hz})$, beta $\beta$ (17.5-35 Hz), low-gamma $\gamma(31-50 \mathrm{~Hz})$ and highgamma $\gamma(51-70 \mathrm{~Hz})$, are introduced. Such band setting is consistent with previous research on MDD [13]. For a specific frequency band, we average the MPWD coefficients from the frequency direction and obtain a new $p$-channel signals. Via setting the channels as nodes, and determining the edges between nodes via PLV, a frequency dependent brain network can be constructed. PLV is a representative method for quantifying the synchronization between EEG signals. Taking $x(t), t=1,2, \ldots, N$ as an example, Hilbert transform is used to decompose $x(t)$ into two parts, i.e., the analytic amplitude $A_{x}(t)$ and the analytic phase $\phi_{x}(t)[45]$ :

$z(t)=x(t)+i \tilde{x}(t)=A_{x}(t) \exp \left(i \phi_{x}(t)\right)$,

where $\tilde{x}(t)$ stands for the Hilbert transform of $x(t)$. According to [29], the mathematical expression of PLV between two times series (e.g. $x(t)$ and $y(t)$ ) is defined as:

$P L V=\frac{1}{N}\left|\sum_{n=1}^{N} e^{i \theta(t)}\right|$,

where $N$ represents the length of the signals, $\theta(t)=$ $\phi_{x}(t)-\phi_{y}(t)$ stands for the instantaneous phase difference. By inferring brain networks in different frequency bands, FDMB network with six layers (corresponding to $\delta, \theta, \alpha, \beta$, low- $\gamma$, and high- $\gamma$, respectively) can be constructed, with $p$ nodes in each layer. 


\subsection{Multilayer deep convolutional neural network}

Here, taking FDMB network as input, a multilayer deep CNN (MDCNN) model is carefully designed for the detection of MDD. Figure. 2 provides the detailed architecture. The multilayer structure of the MDCNN model exactly matches the structure of the FDMB network. Different layers of the MDCNN model share the same structure, and their outputs are finally concatenated together for classification. Such design helps to effectively learn and integrate the rich network topology characteristics hidden in different layers of the FDMB network. Particularly, a core block composed of three consecutive convolutional layers, one batch normalization (BN) layer and one pooling layer is designed as the core of the MDCNN model. Such combination of basic hidden layers can effectively avoid overfitting and speed up model training, and has been confirmed by many existing works.

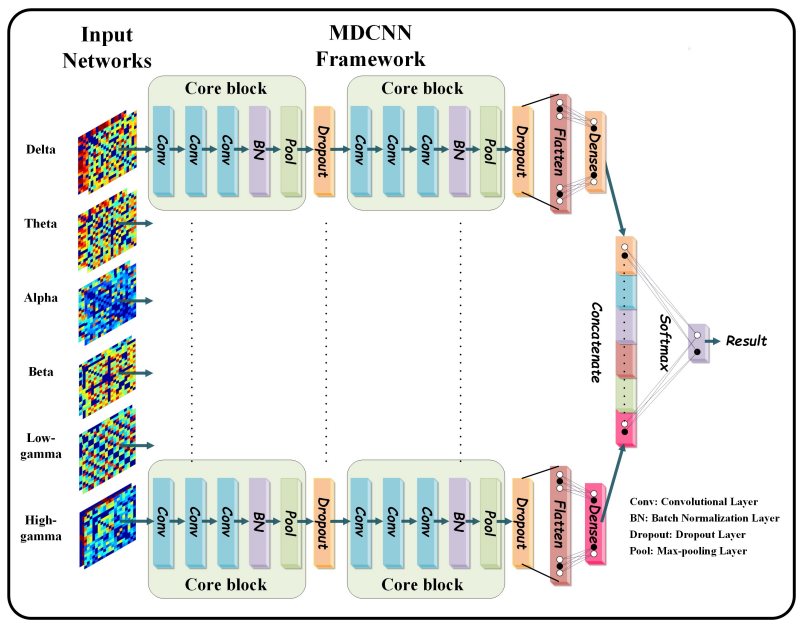

Fig. 2 The architecture of the MDCNN model.

Next, we take the first layer (corresponding to delta band) as an example to specify the design of the model. The detailed parameters are displayed in Table I. As described before, FDMB network integrates the time, frequency and channel-related information, and allows effectively characterizing the multivariate times series of complex systems. Note that, FDMB network, stored in the adjacency matrix, presents rich features via various distributions of network edges and is a grid-like data. Existing studies have confirmed that by setting the appropriate convolution kernel, the convolution layer shows a strong advantage in analyzing grid-like data. So we arrange the convolution layer at the beginning of the core block.

Generally, convolutional layer uses a "kernel" to extract the representative features from the previous layer,
Table 1 The detailed parameters of one layer of the MDCNN model.

\begin{tabular}{lll}
\hline Layers & Parameters & Output \\
\hline Core Block 1 & Conv, 5*5@32* (PRelu) & $19,19,32$ \\
& Conv, 5*5@32 (PRelu) & $19,19,32$ \\
& Conv, 5*5@32 (PRelu) & $19,19,32$ \\
& Batch Normalization & $19,19,32$ \\
& Max-pool, 2*2 & $9,9,32$ \\
\hline \multirow{2}{*}{ Dropout 1 } & Dropout layer, p=0.22 & $9,9,32$ \\
\hline Core Block 2 & Conv, 3*3@64 (PRelu) & $9,9,64$ \\
& Conv, 3*3@64 (PRelu) & $9,9,64$ \\
& Conv, 3*3@64 (PRelu) & $9,9,64$ \\
& Batch Normalization & $9,9,64$ \\
& Max-pool, 2*2 & $4,4,64$ \\
\hline Dropout 2 & Dropout layer, p=0.18 & $4,4,64$ \\
\hline Flatten & Flatten layer & 1024 \\
\hline Dense & Dense layer & 320 \\
& (PRelu, L2 norm =0.00015) \\
\hline
\end{tabular}

* Conv, 5*5@32 means that 32 kernels with $5 * 5$ size in this convolutional layer.

and output them in the form of a "feature map". The $k_{t h}$ feature maps $\left(z_{i, j}\right)_{k}^{l}$ of the $l_{t h}$ layer can be obtained by the following fomula:

$\left(z_{i, j}\right)_{k}^{l}=f\left(\operatorname{conv}\left(w_{k}^{l}, x_{i, j}^{l-1}\right)+b_{k}^{l}\right)$,

where $x_{i, j}^{l-1}$ denotes the input block centered at location $(i, j)$ in the $(l-1)_{t h}$ layer, $w_{k}^{l}$ and $b_{k}^{l}$ represent the weight matrix and bias term of the $k_{t h}$ convolution kernel, respectively, conv (.) means the convolution operation. Same convolution is utilized. After careful research, we set three convolutional layers in each core block, which is deep enough and stable for extracting the effective features. A BN layer is then added after the convolutional layers to accelerate the training process. For a specific layer $l$ with an input $x^{(l)}$, the $\mathrm{BN}$ is implemented as follows:

$y^{(l)}=\gamma^{(l)} \hat{x}^{(l)}+\beta^{(l)}$,

where $\gamma^{(l)}$ and $\beta^{(l)}$ are learnable parameters which aim to keep the distribution of $y^{(l)}$ and $x^{(l)}$ consistent, $\hat{x}^{(l)}$ is the normalized input data with the following form:

$\hat{x}^{(l)}=\frac{x^{(l)}-\operatorname{mean}\left(x^{(l)}\right)}{\operatorname{std}\left(x^{(l)}\right)}$,

where mean $(\cdot)$ and $\operatorname{std}(\cdot)$ mean the expectation and standard deviation of $x^{(l)}$. Finally, a pooling layer is added to achieve shift-invariance from the perspective of downsampling. For each feature map $\left(z_{:,:}\right)_{k}^{l}$, the pooling function can be realized via

$\left(P_{i, j}\right)_{k}^{l+1}=\operatorname{pool}\left(\left(z_{m, n}\right)_{k}^{l}\right), \forall(m, n) \in R_{i j}$,

where $R_{i j}$ means the adjacent region around the location $(i, j)$ and its size is consistent with the filter of the 
pooling layer, and pooling operation $\operatorname{pool}(\cdot)$ is elected as max pooling here. After one core block, a dropout layer is added to avoid over-fitting, which randomly selects the output neurons of the previous layer with a probability $P_{d}$.

Considering the balance between feature extraction ability and computational load, we use two core blocks in one layer of the MDCNN model and their parameter settings are different. Specifically, the convolution kernel of the core block 2 is smaller than core block 1 . After two core blocks, a fully connected layer (dense layer) is added to integrate the learned features. In our work, parametric rectified linear unit (PRelu) is used as the activation function of the convolutional layers and fully connected layers. The PRelu function has the following form:

$g(x)=\left\{\begin{array}{l}x, x>0 \\ \alpha x, \text { otherwise }\end{array}\right.$

where $\alpha$ is a learnable parameter, making the model more learnable and helps to accelerate the training process. Finally, as shown in Fig. 2, all learned features from six inputs of the MDCNN model are concatenated together for classification, using softmax activation function.

\section{MDD dataset}

In this study, MDD dataset contributed by Mumtaz et al. [13] is used, which contains 34 MDD patients and 30 healthy controls. The MDD patients contain 17 males and 17 females with the average age of $40.3 \pm 12.9$ years, while the healthy controls contain 21 males and 9 females with the average age of $38.3 \pm 15.6$ years. According to the Diagnostic and Statistical Manual-IV (DSM-IV) [46], all the MDD patients met the criterion for depression. The human ethics committee of Hospital University Sains Malaysia (HUSM), Malaysia, has agreed on the implementation of the experiment. During the experiment, the resting state EEG data was recorded from each participant, which involved 5-min eyes-closed (EC) condition and 5-min eyes-open (EO) condition. A 19-electrode EEG cap with linked-ear (LE) reference [47] was utilized to capture the EEG signals from the scalp. All electrodes are placed in accordance with international 10 20 electrode placement standard [48]. Nineteen electrodes involve the frontal (Fp1, Fp2, F3, F4, F7, F8, Fpz), temporal (T3, T4, T5, T6), parietal (P3, P4, P7, P8), occipital (O1, O2) and central (C3, C4) areas. The sample rate was $256 \mathrm{~Hz}$. Referring to the existing work on this dataset [10-13], the original EEG signals were filtered by a bandpass filter (0.5-70 $\mathrm{Hz}$ ) to eliminate interference, and the power line noise was eliminated by a $50 \mathrm{~Hz}$ notch filter. It should be noted that not all subjects have both EC and EO data in the available data files. For one file, corresponding to one state (EC or EO) of a subject, we uniformly select the first 180 s of data for analysis.

\section{Results and discussion}

\subsection{MPWD and FDMB network of MDD patients}

Brain is a typical complex system, and its internal activities are closely related to the rhythm information. In order to analyze the brain activities of MDD patients from the time-frequency point of view, MPWD analysis is performed on the EEG signals. Two EEG signal segments are selected, with a length of 10s, corresponding to an MDD patient and a healthy control, respectively. The EEG signals and corresponding MPWD are shown in Fig. 3. As can be seen, there are significant differences in MPWD between the MDD patient and healthy control. In detail, the MPWD of MDD patient presents a more scattered distribution. Some short-term concentrated distributions appear in the low frequency band.

Based on the multilayer network architecture, FDMB network integrates the time, frequency and channelrelated information, and allows effectively characterizing the brain. Two randomly selected FDMB networks are visualized in Fig. 4. One sub-figure corresponds to one layer of the FDMB network and is related to one specific frequency band. FDMB network of MDD patient is on the first row, and FDMB network of healthy control is on the second row. As can be seen, the network topologies show obvious differences in different frequency bands. This suggests that MDD is closely related to band energy, which is consistent with the conclusions of existing work [49]. All these suggest that the FDMB network has the ability to characterize the differences of the brain between MDD patients and healthy controls, and confirm the importance of rhythms and electrodes (channels) in the study of MDD.

\subsection{MDCNN model for classification}

Based on the MDD dataset described in Section. III, a sample set is constructed. For each subject, the first 180 s of data are selected. A non-overlapping sliding window with length $2 \mathrm{~s}$ is used to partition the samples. In total, there are 5130 samples in the healthy control group and 5580 samples in the MDD patient group. Ten-fold cross-validation is utilized. For each fold, 90\% of the whole samples are chosen as the training set and 

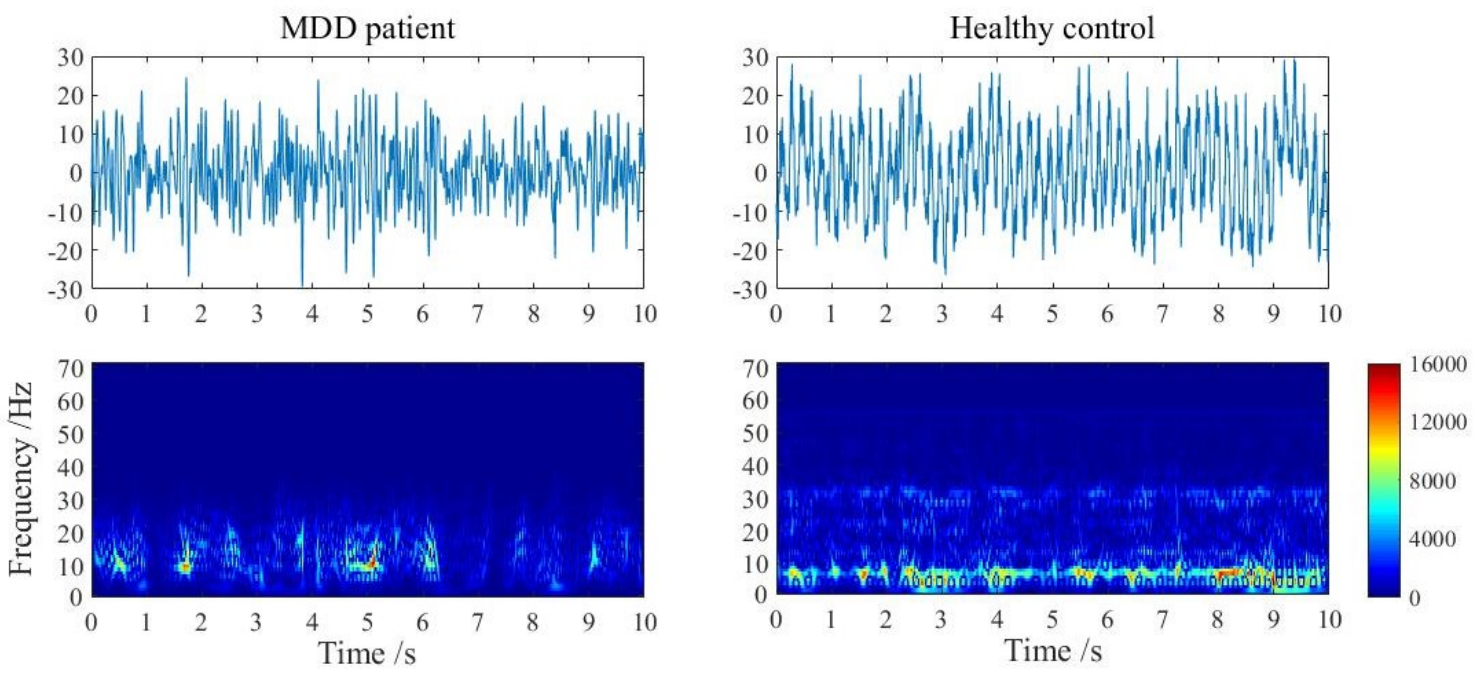

Fig. 3 MPWD analysis results for an MDD patient and a healthy control. The first row is the EEG signals from one randomly selected channel. The second row shows the time-frequency distribution of the EEG signals.
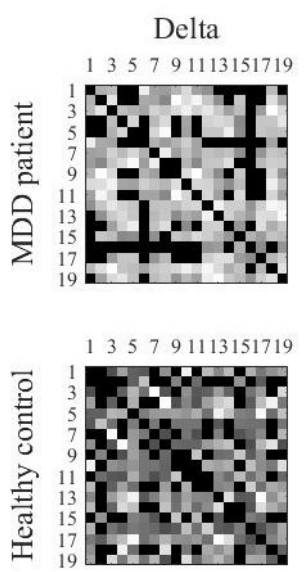

Theta
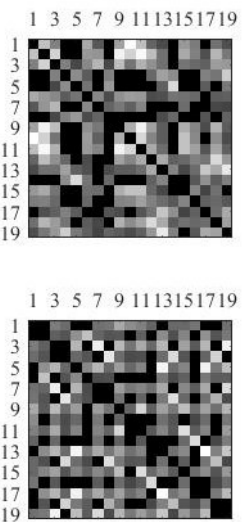

Alpha
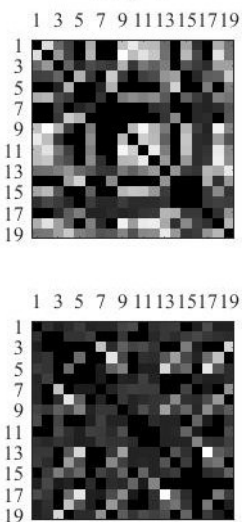

Beta
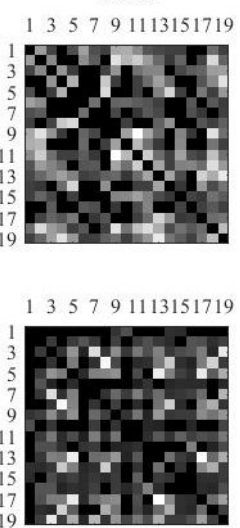

Low-gamma
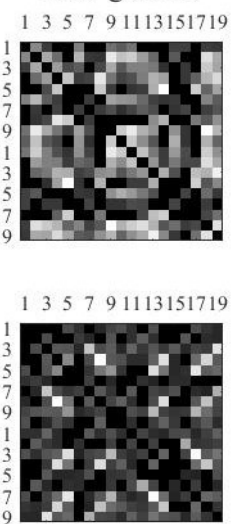

High-gamma
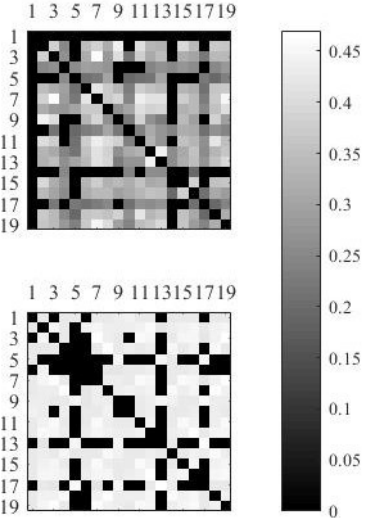

Fig. 4 Visualization of brain networks in different frequency bands of an MDD patient and a healthy control. The color represents the degree of connection between different channels.

the remaining $10 \%$ as the testing set. We also selected $10 \%$ of the training samples as the validation set. Taking the FDMB networks as input, the MDCNN model is trained using Keras through a fully-supervised way. Based on the Adam optimizer, all kinds of weights and biases are optimized via minimizing the cross-entropy loss function. The best model is recorded when the loss on the validation set reaches a minimum value. All these are performed on a server with a $4.20 \mathrm{GHz}$ Intel(R) Core(TM) i7-7700K CPU, a NVIDIA GeForce GTX TITAN X and 16G RAM. Learning rate is set as 0.0001. 300 epochs are conducted with batch size 128 . Four performance metrics, including accuracy, sensitivity, specificity, and F-measure, are introduced to evaluate the model's performance on the testing set. They are defined as follows:

$$
\text { Accuracy }=\frac{T P+T N}{T P+T N+F P+F N},
$$

Sensitivity $=\frac{T P}{T P+F N}$

Specificity $=\frac{T N}{T N+F P}$,

$F-$ measure $=\frac{2 \times T P}{F P+F N+2 \times T P}$,

where TP, TN, FP and FN mean "true positive", "true negative", "false positive" and "false negative", respectively. Among them, F-measure can be regarded as a weighted harmonic average of sensitivity and specificity. The classification results of our method and existing works on this MDD dataset (Mumtaz et al. [13]) are displayed in Table. II. As can be seen, our method performs well on all performance metrics, with accuracy $97.27 \%$, sensitivity $97.22 \%$, specificity $97.35 \%$, Fmeasure 0.97 . 
Table 2 Classification results of our work and some existing works on this MDD dataset.

\begin{tabular}{|c|c|c|c|c|c|c|}
\hline \multirow{2}{*}{ Study } & \multirow{2}{*}{ Methods or Features } & \multirow{2}{*}{ Classifier } & \multicolumn{4}{|c|}{ Performance* } \\
\hline & & & $\mathrm{ACC}$ & SEN & SPE & F-M \\
\hline Mumtaz et al.(2017) [13] & Wavelet features & Logistic regression & $87.50 \%$ & $95 \%$ & $80 \%$ & 0.81 \\
\hline Mahato et al.(2019) [10] & Alpha power + RWE & MLPNN; RBFN & $93.33 \%$ & $94.44 \%$ & $87.78 \%$ & \\
\hline Mumtaz et al.(2017) [12] & SL, coherence and MI & SVM & $94.70 \%$ & $98.30 \%$ & $91.40 \%$ & 0.95 \\
\hline Our work & FDMB+MDCNN & MDCNN & $97.27 \%$ & $97.22 \%$ & $97.35 \%$ & 0.97 \\
\hline
\end{tabular}

* The ACC, SEN, SPE, F-M in the Performance column refers to accuracy, sensitivity, specificity and F-measure, respectively.

In order to further explore the impact of different frequency bands on MDD detection, we also provide the results when using only one single frequency band. Particularly, we regard $0.5-70 \mathrm{~Hz}$ as one frequency band, which means no band division and the classification results in this case are also tested. The corresponding results are shown in Fig. 5. By fusing rich information

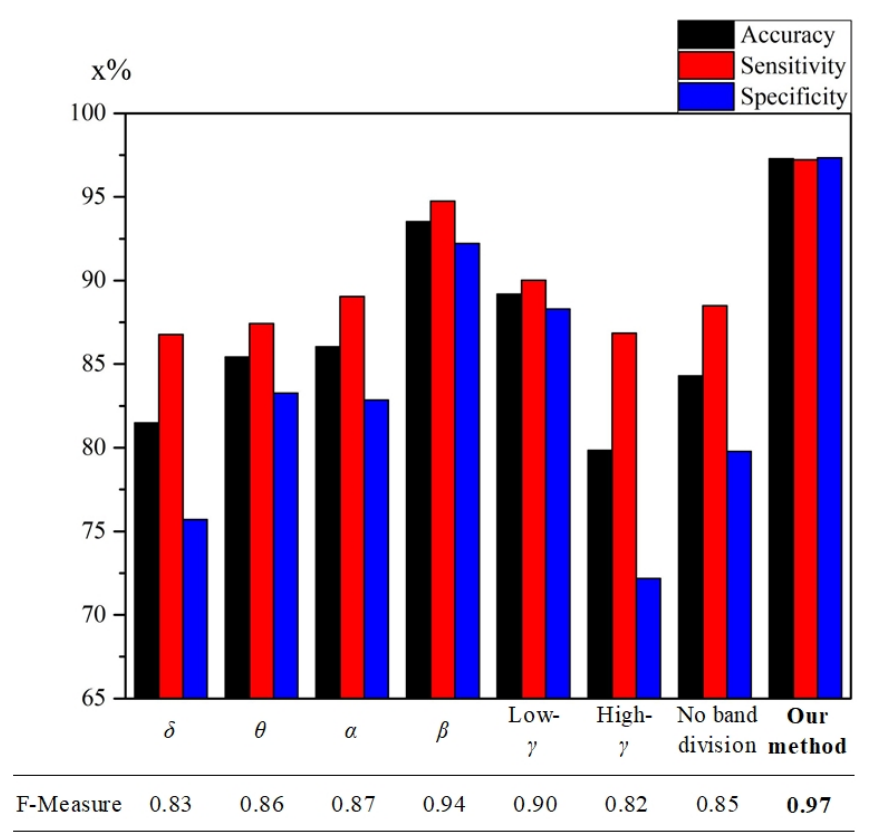

Fig. 5 The classification results by using different frequency bands.

from multiple frequency bands, our method combining multilayer brain network and deep learning shows the best results. And the results of the beta and low-gamma bands are significantly higher than other bands, indicating that more information can be obtained from these two bands to detect MDD. It is worth mentioning that when no band division is performed, the classification accuracy is only $84.31 \%$. This is because that the characteristics in different frequency bands cannot be specifically used, which causes confusion of information.

In addition, we plot the receiver operating characteristics (ROC) curve of different situations, shown in Fig. 6. And the area under curve (AUC) is calculated. AUC is a commonly used measure for illustrating the performance of a binary classifier system. The AUC value lies between 0 and 1 , and a higher AUC value indicates a better classification performance. We can see that the AUC ranking is roughly the same as accuracy ranking. Our method has the highest AUC of 0.997 , showing that the combined FDMB+MDCNN framework using all frequency bands has a good classification ability. We also test the sensitivity of the core

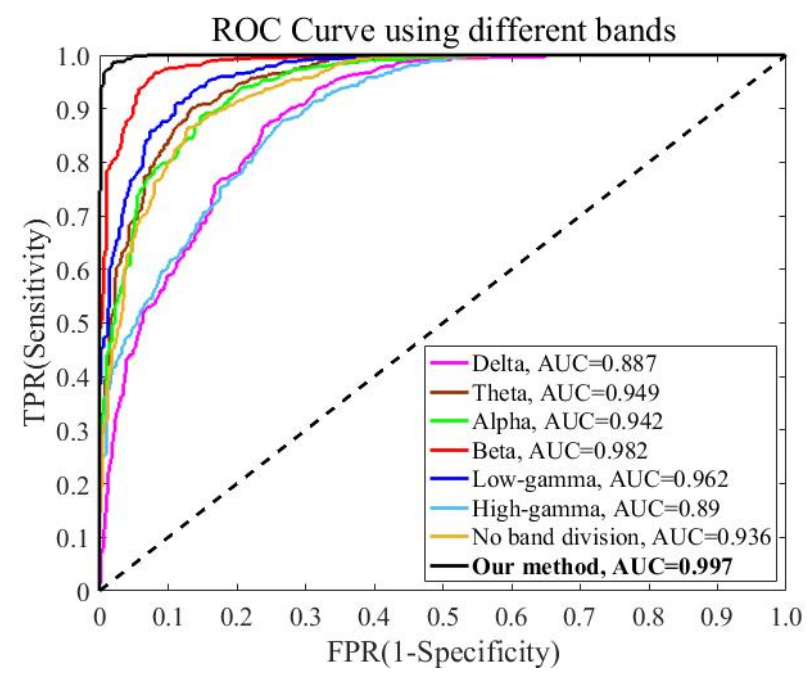

Fig. 6 The ROC curves of the classification using different frequency bands.

block to the number of convolutional layers, as shown in Table III. We find that when we arrange three convolutional layers in one core block, the proposed MDCNN model achieves the best classification accuracy. And the results are not greatly dependent on the number of convolutional layers in the core block. All these demonstrate that the proposed framework combining multilayer brain network and MDCNN model can learn effective information for MDD detection. 
Table 3 Classification results using different numbers of convolutional layers in the core block.

\begin{tabular}{ccccc}
\hline $\begin{array}{c}\text { Number of } \\
\text { conv layers* }\end{array}$ & ACC & SEN & SPE & F-M \\
\hline 1 & $96.94 \%$ & $96.02 \%$ & $97.93 \%$ & $97.03 \%$ \\
2 & $97.23 \%$ & $97.47 \%$ & $96.96 \%$ & $97.34 \%$ \\
3 & $97.27 \%$ & $97.22 \%$ & $97.33 \%$ & $97.38 \%$ \\
\hline
\end{tabular}

* "conv layers" means convolutional layers. "ACC", "SEN", "SPE", "F-M" refer to accuracy, sensitivity, specificity and F-measure, respectively.

\section{Conclusion}

MDD severely affects the daily lives of patients and their families, and is widespread worldwide. Efficient detection of MDD can provide the necessary guidance for timely treatment and reduce the adverse effects of the disease. Here, based on EEG signals, a novel FDMB network with multilayer structure is developed. FDMB network integrates the time, frequency and channelrelated information, and allows effectively characterizing the brain of MDD. From the network view, it can be found that the network topologies of the FDMB networks show obvious differences for MDD patients and healthy controls, in different frequency bands. Further, aiming at fully extracting the effective features from the FDMB network, a matched multilayer deep CNN (MDCNN) model is designed. The multilayer structure of the MDCNN model exactly matches the structure of the FDMB network. By comparison, we find that our framework combining brain network and deep CNN can effectively distinguish between MDD patients and healthy controls. This also illustrates the effectiveness of deep learning theory in EEG signal analysis. Considering the universality and validity of our method, we expect it to be successfully applied in more EEG signal classification tasks.

Acknowledgements This work was supported in part by the National Natural Science Foundation of China under Grants Nos. 61922062 and 61873181.

\section{Conflict of interest}

The authors declare that they have no conflict of interest.

\section{References}

1. R. C. Kessler, P. Berglund, O. Demler, et al.: The epidemiology of major depressive disorder: results from the National Comorbidity Survey Replication (NCS-R). JAMAJ. Am. Med. Assoc. 289, 3095-105 (2003).
2. D. S. Hasin, R. D. Goodwin, F. S. Stinson, and B. F. Grant: Epidemiology of major depressive disorder: results from the National Epidemiologic Survey on alcoholism and related conditions. Arch. Gen. Psychiatry 62, 1097-1106 (2005)

3. D. D. Xu, W. W. Rao, X. L. Cao, et al.: Prevalence of major depressive disorder in children and adolescents in China: A systematic review and meta-analysis. J. Affect. Disord. 241, 592-598 (2018)

4. A. H. Miller, and C. L. Raison: The role of inflammation in depression: from evolutionary imperative to modern treatment target. Nat. Rev. Immunol. 16, 22-34 (2016)

5. B. I. Goldstein, W. Shamseddeen, A. Spirito, et al.: Substance use and the treatment of resistant depression in adolescents. J. Am. Acad. Child Psy. 48, 1182-1192 (2009)

6. K. D. Young, G. J. Siegle, V. Zotev, R. Phillips, M. Misaki, H. Yuan, W. C. Drevets, and J. Bodurka: Randomized clinical trial of real-time fMRI amygdala neurofeedback for major depressive disorder: effectson symptoms and autobiographical memory recall. Am. J. Psychiat. 174, 748-755 (2017)

7. B. W. Dunlop, J. K. Rajendra, W. E. Craighead, et al.: Functional connectivity of the subcallosal cingulate cortex and differential outcomes to treatment with cognitivebehavioral therapy or antidepressant medication for major depressive disorder. Am. J. Psychiat. 174, 533-545 (2017)

8. I. M. Lin, S. Y. Fan, C. F. Yen, et al.: Heart rate variability biofeedback increased autonomic activation and improved symptoms of depression and insomnia among patients with major depression disorder. Clin. Psychopharm. Neu. 17, 222-232 (2019)

9. A. Mestanikova, M. Mestanik, I. Ondrejka, et al.: Complex cardiac vagal regulation to mental and physiological stress in adolescent major depression. J. Affect. Disord. 249, 234$241(2019)$

10. S. Mahato, and S. Paul: Detection of major depressive disorder using linear and non-linear features from EEG signals. Microsyst. Technol. 25, 1065-1076 (2018)

11. W. Mumtaz, L. Xia, S. S. A. Ali, M. A. M. Yasin, M. Hussain, and A. S. Malik: Electroencephalogram (EEG)based computer-aided technique to diagnose major depressive disorder (MDD). Biomed. Signal Proces. 31, 108-115 (2017)

12. W. Mumtaz, S. S. A. Ali, M. A. M. Yasin, and A. S. Malik: A machine learning framework involving EEG-based functional connectivity to diagnose major depressive disorder (MDD). Med. Biol. Eng. Comput. 56, 233-246 (2018)

13. W. Mumtaz, L. Xia, M. A. Mohd Yasin, S. S. Azhar Ali, and A. S. Malik: A wavelet-based technique to predict treatment outcome for major depressive disorder. PLoS One 12, e0171409 (2017)

14. D. A. Pizzagalli, C. A. Webb, D. G. Dillon, et al.: Pretreatment rostral anterior cingulate cortex theta activity in relation to symptom improvement in depression: a randomized clinical trial. JAMA Psychiat. 75, 547-554 (2018)

15. M. Ahmadlou, H. Adeli, and A. Adeli: Fractality analysis of frontal brain in major depressive disorder. Int. J. Psychophysiol. 85, 206-211 (2012)

16. J. R. Goldschmied, P. Cheng, R. Armitage, and P. J. Deldin: A preliminary investigation of the role of slowwave activity in modulating waking EEG theta as a marker of sleep propensity in major depressive disorder. J. Affect. Disorders 257, 504-509 (2019)

17. O. W. Murphy, K. E. Hoy, D. Wong, N. W. Bailey, P. B. Fitzgerald, and R. A. Segrave: Individuals with depression display abnormal modulation of neural oscillatory activity 
during working memory encoding and maintenance. Biol. Psychol., 107766 (2019)

18. R. B. Pachori, and P. Sircar: EEG signal analysis using FB expansion and second-order linear TVAR process. Signal Process. 88, 415-420 (2008)

19. M. Sharma, D. Goyal, P. V. Achuth, and U. R. Acharya: An accurate sleep stages classification system using a new class of optimally time-frequency localized three-band wavelet filter bank. Comput. Biol. Med. 98, 58-75 (2018)

20. F. Riaz, A. Hassan, S. Rehman, I. K. Niazi, and K. Dremstrup: EMD-based temporal and spectral features for the classification of eeg signals using supervised learning. IEEE Trans. Neural Syst. Rehabil. Eng. 24, 28-35 (2016)

21. G. L. Tian, and Y. Liu: Simple convolutional neural network for left-right hands motor imagery eeg signals classification. International Journal of Cognitive Informatics and Natural Intelligence 13, 36-49 (2019)

22. A. Bhattacharyya, M. Sharma, R. B. Pachori, P. Sircar, and U. R. Acharya: A novel approach for automated detection of focal EEG signals using empirical wavelet transform. Neural Comput. Appl. 29, 47-57 (2018)

23. A. Ahrabian, D. Looney, L. Stankovic, and D. P. Mandic: Synchrosqueezing-based time-frequency analysis of multivariate data. Signal Process. 106, 331-341, (2015)

24. G. V. L. de Lima, P. T. M. Saito, F. M. Lopes, and P. H. Bugatti: Classification of texture based on bag-of-visualwords through complex networks. Expert Syst. Appl. 133, 215-224, (2019)

25. J. Li, H. Jiang, Z. Yu, and C. Hu: Dynamical analysis of rumor spreading model in homogeneous complex networks. Appl. Math. Comput. 359, 374-385, (2019)

26. H. Wang, J. Wang, M. Small, and J. M. Moore: Review mechanism promotes knowledge transmission in complex networks. Appl. Math. Comput. 340, 113-125, (2019)

27. F. C. Morabito, M. Campolo, D. Labate, et al.: A longitudinal EEG study of Alzheimer's disease progression based on a complex network approach. Int. J. Neural Syst. 25, 1550005 (2015)

28. Z. Gao, W. Dang, M. Liu, W. Guo, K. Ma and G. Chen: Classification of EEG signals on VEP-based BCI systems with broad learning. IEEE Trans. Syst., Man, Cybern., Syst. 2019, doi:10.1109/TSMC.2020.2964684

29. J. P. Lachaux, E. Rodriguez, J. Martinerie, and F. J. Varela: Measuring phase synchrony in brain signals. Hum. Brain Mapp. 8, 194-208 (1999)

30. M. Wang, H. El-Fiqi, J. K. Hu, and H. A. Abbass: Convolutional neural networks using dynamic functional connectivity for EEG-based person identification in diverse human states. IEEE Trans. Inf. Forensic Secur. 14, 3259$3272(2019)$

31. A. Sole-Ribalta, M. De Domenico, S. Gomez, and A. Arenas: Random walk centrality in interconnected multilayer networks. Physica D 323, 73-79 (2016)

32. S. Martincic-Ipsic, D. Margan, and A. Mestrovic: Multilayer network of language: A unified framework for structural analysis of linguistic subsystems. Physica A 457, 117$128(2016)$

33. W. D. Dang, Z. K. Gao, D. M. Lv, M. X. Liu, Q. Cai, and X. L. Hong: A novel time-frequency multilayer network for multivariate time series analysis. New J. Phys. 20, 125005 (2018)

34. F. D. Sahneh, C. Scoglio, and P. Van Mieghem: Generalized epidemic mean-field model for spreading processes over multilayer complex networks. IEEE-ACM Trans. Netw. 21, 1609-1620 (2013)

35. Z. Gao, W. Dang, C. Mu, Y. Yang, S. Li, and C. Grebogi: A novel multiplex network-based sensor information fusion model and its application to industrial multiphase flow system. IEEE Trans. Ind. Inform. 14, 3982-3988 (2018)

36. Z. Gao, S. Zhang, W. Dang, S. Li, and Q. Cai: Multilayer network from multivariate time series for characterizing nonlinear flow behavior. Int. J. Bifurcat. Chaos 27, 1750059 (2017)

37. J. M. Buldu, and M. A. Porter: Frequency-based brain networks: From a multiplex framework to a full multilayer description. Netw. Neurosci. 2, 418-441, (2018)

38. E. Lin, P. H. Kuo, Y. L. Liu, Y. W. Y. Yu, A. C. Yang, and S. J. Tsai: A deep learning approach for predicting antidepressant response in major depression using clinical and genetic biomarkers. Front. Psychiatry 9, (2018)

39. K. Zhang, W. Zuo, Y. Chen, D. Meng, and L. Zhang: Beyond a gaussian denoiser: residual learning of deep CNN for image denoising. IEEE Trans. Image Process. 26, 31423155 (2017)

40. V. Badrinarayanan, A. Kendall, and R. Cipolla: SegNet: a deep convolutional encoder-decoder architecture for image segmentation. IEEE Trans. Pattern Anal. Mach. Intell. 39, 2481-2495 (2017)

41. W. D. Dang, Z. K. Gao, L. H. Hou, D. M. Lv, S. M. Qiu, and G. R. Chen: A novel deep learning framework for industrial multiphase flow characterization. IEEE Trans. Ind. Inform. 15, 5954-5962 (2019)

42. C. Szegedy, S. Ioffe, V. Vanhoucke, A. A. Alemi, and Aaai, Inception-v4, inception-ResNet and the impact of residual connections on learning," Proc. AAAI Conf. Artif. Intell. (2017)

43. Z. K. Gao, X. M. Wang, Y. X. Yang, C. X. Mu, Q. Cai, W. D. Dang, and S. Y. Zuo: EEG-based spatio-temporal convolutional neural network for driver fatigue evaluation. IEEE Trans. Neural Netw. Learn. Syst. 30, 2755-2763 (2019)

44. J. M. Lilly, and S. C. Olhede: Analysis of modulated multivariate oscillations. IEEE Trans. Signal Process. 60, 600$612(2012)$

45. M. H. Myers, A. Padmanabha, G. Hossain, A. L. de Jongh Curry, and C. D. Blaha: Seizure prediction and detection via phase and amplitude lock values. Front. Hum. Neurosci. 10, 80 (2016)

46. A. P. Association, Diagnostic and statistical manual of mental disorders (DSM-5): American Psychiatric Pub, (2013)

47. J. J. B. R. M. Dien: Instruments, and computers, issues in the application of the average reference: review, critiques, and recommendations. Behav. Res. Methods Instr. Comput. vol. 30, 34-43 (1998)

48. H. H. J. E. C. N. Jasper: The ten-twenty electrode system of the international federation. Neurophysiology 10, 370375 (1958)

49. S. C. Roh, E. J. Park, M. Shim, and S. H. Lee: EEG beta and low gamma power correlates with inattention in patients with major depressive disorder. J. Affect. Disord. 204, 124-130 (2016) 